\title{
Proteomic Signature of Urosepsis: From Discovery in a Rabbit Model to Validation in Humans
}

Guangju Ge ${ }^{1, \ddagger}$, Qiming Zheng ${ }^{1, \ddagger}$, Zeyu Sun ${ }^{2, \ddagger}$, Huan Wang $^{1}$, Huailan Wang ${ }^{1}$, Keyi

Ren ${ }^{2}$, Haiyang $\mathrm{Wu}^{1}$, Shibin $\mathrm{Zhu}^{1}$, Gonghui $\mathrm{Li}^{1,}$ *

1 Departments of Urology, Sir Run Run Shaw Hospital, School of Medicine, Zhejiang University, Hangzhou, Zhejiang, 310016, China.

2 State Key Laboratory for Diagnosis and Treatment of Infectious Diseases, National Clinical Research Center for Infectious Diseases, Collaborative Innovation Center for Diagnosis and Treatment of Infectious Disease, The First Affiliated Hospital, School of Medicine, Zhejiang University, Hangzhou, Zhejiang, 310003, China.

*Gonghui Li: 3193119@zju.edu.cn 


\section{Table of content}

1. Detailed information of targeted proteomic (S-3)

2. Inclusion and exclusion criteria (S-5)

3. Supplemental tables of: (S-6)

(1) clinical characteristics of patients; (S-6)

(2) urine WBC, urine culture, organ dysfunction and quick SOFA; (S-7)

(3) 58 peptides derived from 20 candidates for the targeted proteomics; (S-9)

(4) 12 proteins successfully quantified in targeted proteomics; (S-13)

(5) 15 co-eluted and co-fragmented proteins in targeted proteomics; (S-14)

(6) Identification statistics for consensus database searching strategy; (S-15)

(7) 9 potential urosepsis diagnostic biomarkers with auROC $>0.7$; (S-16)

(8) Logistic model with 4 proteins and its statistics; (S-17)

(9) Logistic model with 3 proteins and its statistics; (S-17)

(10)Kidney injury associated DEPs in the discovery TMT datasets. (S-18) 


\section{Supplementary}

\section{Targeted proteomics.}

To build the MS/MS spectra library for reference, a small set of samples $(n=10)$ were first analyzed using LC-MSMS under the DDA scheme with extensive HPRP fractionation. Peptides ( $\sim 500 \mathrm{ng})$ were reconstituted in phase $\mathrm{A}(2 \% \mathrm{ACN}, 0.1 \% \mathrm{FA})$ and were separated by an Acclaim ${ }^{\mathrm{TM}}$ PepMap $100 \mathrm{C} 18\left(15 \mathrm{~cm}^{*} 0.075 \mathrm{~mm}\right)$ nanoRPLC column on an U3000 nanoUPLC system (ThermoFisher) at a flow rate of $400 \mathrm{~nL} / \mathrm{min}$. The gradient started with $3 \%$ of mobile phase B ( $98 \%$ ACN, $0.1 \%$ FA) and increased to $5 \%$ in $2 \mathrm{~min}$, then reached $18 \% \mathrm{~B}$ in $64 \mathrm{~min}$ and $32 \% \mathrm{~B}$ in $20 \mathrm{~min}$. The gradient finally reached $80 \% \mathrm{~B}$ in $10 \mathrm{~min}$ and was then held for $10 \mathrm{~min}$ before it returned to $3 \%$ $\mathrm{B}$ in $1 \mathrm{~min}$ and kept for 9 min re-equilibration until next injection. The total analysis time per injection was $116 \mathrm{~min}$. The nanoLC was coupled to an Orbitrap Q-Exactive HFX mass spectrometer (Thermo). The source was operated at $2.2 \mathrm{kV}$. The DDA analysis included a full MS survey scan from 350 to 1,500 Th at the resolution of 120,000 FWHM (at m/z 200) with automatic gain control (AGC) Th target set to 3e6, followed by 20 most intense peaks were selected for fragmentation by higher-energy collision dissociation (HCD). The MS/MS spectra were acquired with 15,000 FWHM resolution with AGC set to 5e4. Search results from MaxQuant (v1.6.1.0) against the human Uniprot database were loaded to Skyline (v4.1.0.18169) for selection of peptide ions according to the targeted proteomic workflow protocol described previously. ${ }^{1}$

A list of 58 peptides derived from 20 protein markers (Table S1) was generated for the targeted proteomic survey. Thereafter, all samples were analyzed using the same chromatographic conditions while the MS operated under the parallel-reaction monitoring (PRM) acquisition scheme, which included a full MS survey scan from 350 to 1,300 at the resolution of $45,000 \mathrm{FWHM}$ (at m/z 200) with AGC set to $3 \mathrm{e} 6$, followed by HCD events to collect MS/MS spectra scheduled for a list of pre-defined 58 peptide targets. The isolation window was set to $1 \mathrm{Th}$. All MS/MS spectra were acquired at the resolution of 30,000 FWHM with AGC set to 5e5. The HCD 
normalized collision energy was set to $27 \%$.

1. Sherrod SD, Myers MV, Li M, Myers JS, Carpenter KL, Maclean B, et al. Label-free quantitation of protein modifications by pseudo selected reaction monitoring with internal reference peptides. J Proteome Res. 2012; 11: 3467-79. 


\section{Inclusion and exclusion criteria:}

Inclusion criteria:

1) Received UUTEL in our center;

2) Blood samples should be drawn 2 hours after UUTEL;

3) The inclusion time is limited to June 2017 to September 2018;

4) The information and results of laboratory tests and imaging examinations of patients are complete.

Exclusion criteria:

1) Age under 18 years;

2) Existed urosepsis before UUTEL;

3) Complicated with acute trauma or infection in other systems;

4) Complicated with other serious diseases of heart, brain, liver and lung;

5) Complicated with malignant tumor, autoimmune disease or hematological disease. 
Table S1. Clinical characteristics of both patient groups in the validation study

\begin{tabular}{|c|c|c|}
\hline Characteristic & Control $(n=21)$ & Sepsis $(n=20)$ \\
\hline \multicolumn{3}{|l|}{ Basic information } \\
\hline Age (yrs.) & $54.19 \pm 14.92$ & $56.4 \pm 12.2$ \\
\hline Gender (female/male) & $9 / 12$ & $11 / 9$ \\
\hline BMI & $24.28 \pm 4.55$ & $23.93 \pm 4.05$ \\
\hline Heart rate (bpm) & $77.71 \pm 9.46$ & $98.55 \pm 19.87$ \\
\hline Respiratory rate (tpm) & $18.57 \pm 0.88$ & $22.6 \pm 2.67$ \\
\hline Temperature $\left({ }^{\circ} \mathrm{C}\right)$ & $36.55 \pm 0.36$ & $38.56 \pm 0.8$ \\
\hline Hospital stay (days) & $6.95 \pm 5.25$ & $9.2 \pm 5.1$ \\
\hline Surgery time (mins) & $73.1 \pm 23.45$ & $72 \pm 38.94$ \\
\hline \multicolumn{3}{|l|}{ Laboratory parameters } \\
\hline \multicolumn{3}{|c|}{ Preoperative/non-infection period } \\
\hline White blood cell $\left(10^{\wedge} 9 / \mathrm{L}\right)$ & $6.91 \pm 1.67$ & $6.95 \pm 1.47$ \\
\hline Hemoglobin $(g / L)$ & $137.42 \pm 15.05$ & $121.8 \pm 18.06$ \\
\hline Platelet $\left(10^{\wedge} 9 / \mathrm{L}\right)$ & $230.95 \pm 69.29$ & $229.2 \pm 77.51$ \\
\hline $\mathrm{C}$ reactive protein $(\mathrm{mg} / \mathrm{L})$ & $3.03 \pm 3.86$ & $20.02 \pm 17.57$ \\
\hline Pro-calcitonin (ng/mL) & N/A & N/A \\
\hline Albumin $(\mathrm{g} / \mathrm{L})$ & $43.59 \pm 3.7$ & $40.16 \pm 4.34$ \\
\hline \multicolumn{3}{|l|}{ Postoperative/sepsis period } \\
\hline White blood cell $\left(10^{\wedge} 9 / \mathrm{L}\right)$ & $7.68 \pm 2.25$ & $15.43 \pm 4.13$ \\
\hline Hemoglobin $(g / L)$ & $126.14 \pm 18.62$ & $114.9 \pm 18.93$ \\
\hline Platelet $\left(10^{\wedge} 9 / \mathrm{L}\right)$ & $201.04 \pm 66.28$ & $222.8 \pm 101.8$ \\
\hline $\mathrm{C}$ reactive protein $(\mathrm{mg} / \mathrm{L})$ & $5.83 \pm 15.34$ & $98.44 \pm 49.87$ \\
\hline Pro-calcitonin (ng/mL) & N/A & $13.76 \pm 11.39$ \\
\hline Albumin $(\mathrm{g} / \mathrm{L})$ & $38.38 \pm 2.95$ & $32.11 \pm 4.74$ \\
\hline
\end{tabular}


Table S2. urine WBC, urine culture, organ dysfunction and quick SOFA in validation study

\begin{tabular}{|c|c|c|c|c|c|}
\hline Group & ID & $\begin{array}{l}\text { urine WBC / } \\
\text { HP }\end{array}$ & urine culture & $\begin{array}{l}\text { organ } \\
\text { dysfunction }\end{array}$ & $\begin{array}{l}\text { Quick } \\
\text { SOFA }\end{array}$ \\
\hline \multirow{21}{*}{ non-sepsis } & $\mathrm{C} 1$ & negative & not done & none & 0 \\
\hline & $\mathrm{C} 2$ & $1+(11.9)$ & filamentous fungi & none & 0 \\
\hline & C3 & $3+(313.9)$ & P. mirabilis & none & 0 \\
\hline & $\mathrm{C} 4$ & $1+(7.7)$ & $\begin{array}{l}\text { mixed bacterial } \\
\text { flora }\end{array}$ & none & 0 \\
\hline & $\mathrm{C} 5$ & $2+(306.0)$ & negative & none & 0 \\
\hline & C6 & $1+(10.6)$ & negative & none & 0 \\
\hline & $\mathrm{C} 7$ & $3+(92.0)$ & not done & none & 0 \\
\hline & $\mathrm{C} 8$ & $1+(11.5)$ & E.coli & none & 0 \\
\hline & C9 & $1+(30.1)$ & negative & none & 0 \\
\hline & $\mathrm{C} 10$ & $3+(35.5)$ & negative & none & 0 \\
\hline & $\mathrm{C} 11$ & $1+(28.6)$ & negative & none & 0 \\
\hline & $\mathrm{C} 12$ & $1+(78.7)$ & negative & none & 0 \\
\hline & $\mathrm{C} 13$ & $3+(195.1)$ & not done & none & 0 \\
\hline & $\mathrm{C} 14$ & negative & negative & none & 0 \\
\hline & $\mathrm{C} 15$ & $1+(11.9)$ & P. mirabilis & none & 0 \\
\hline & $\mathrm{C} 16$ & $1+(11.9)$ & not done & none & 0 \\
\hline & $\mathrm{C} 17$ & $1+(11.2)$ & C.albicans & none & 0 \\
\hline & $\mathrm{C} 18$ & $3+(17.6)$ & negative & none & 0 \\
\hline & C19 & $1+(5.0)$ & negative & none & 0 \\
\hline & $\mathrm{C} 20$ & negative & not done & none & 0 \\
\hline & $\mathrm{C} 21$ & $2+(5.2)$ & negative & none & 0 \\
\hline \multirow{16}{*}{ sepsis } & S1 & $3+(447)$ & E.coli & none & 0 \\
\hline & S2 & $2+(17.8)$ & negative & none & 1 \\
\hline & S3 & $2+(66.8)$ & E.faecalis & none & 1 \\
\hline & S4 & $3+(262.8)$ & negative & none & 1 \\
\hline & S5 & $2+(16.8)$ & E.faecalis & none & 0 \\
\hline & S6 & $3+(541.4)$ & E.coli & none & 0 \\
\hline & S7 & $3+(54.4)$ & negative & none & 0 \\
\hline & S8 & $3+(65.2)$ & negative & none & 1 \\
\hline & S9 & $3+(50.2)$ & E.faecalis & none & 1 \\
\hline & S10 & $3+(54.5)$ & E.coli & none & 0 \\
\hline & S11 & $3+(28.5)$ & E.coli & none & 1 \\
\hline & S12 & $2+(86.6)$ & C.albicans & none & 1 \\
\hline & S13 & $2+(36.5)$ & negative & none & 1 \\
\hline & S14 & $3+(272.0)$ & P. mirabilis & none & 1 \\
\hline & S15 & $3+(31.5)$ & negative & none & 0 \\
\hline & S16 & $3+(1063.2)$ & E.coli & respiratory & 2 \\
\hline
\end{tabular}


failure,

liver failure,

renal failure

\begin{tabular}{lllll} 
S17 & $2+(88.7)$ & negative & none & 0 \\
S18 & $2+(89.1)$ & $\begin{array}{l}\text { mixed bacterial } \\
\text { flora }\end{array}$ & none & 0 \\
S19 & $2+(99.7)$ & negative & none & 1 \\
S20 & $3+(273.6)$ & negative & none & 0 \\
\hline
\end{tabular}


Table S3. 58 peptides derived from 20 candidates for the targeted proteomics

\begin{tabular}{|c|c|c|c|c|c|}
\hline $\begin{array}{l}\text { Mass } \\
{[\mathrm{m} / \mathrm{z}]}\end{array}$ & $\begin{array}{l}\text { Start } \\
{[\min ]}\end{array}$ & $\begin{array}{l}\text { End } \\
{[\min ]}\end{array}$ & Peptide & protein & \\
\hline 503.2536 & 12.351 & 32.351 & ETWTANVGK & Prothrombin & \\
\hline 437.7427 & 18.249 & 38.249 & VTGWGNLK & Prothrombin & \\
\hline 595.2931 & 32.028 & 52.028 & YGFYTHVFR & Prothrombin & \\
\hline 781.3677 & 44.737 & 64.737 & TATSEYQTFFNPR & Prothrombin & \\
\hline 597.8037 & 46.046 & 66.046 & ELLESYIDGR & Prothrombin & \\
\hline 707.3981 & 42.541 & 62.541 & WILTAAHTLYPK & $\begin{array}{l}\text { Complement } \\
\text { subcomponent }\end{array}$ & $\mathrm{C} 1 \mathrm{r}$ \\
\hline 575.3188 & 58.794 & 78.794 & VLNYVDWIK & $\begin{array}{l}\text { Complement } \\
\text { subcomponent }\end{array}$ & $\mathrm{C} 1 \mathrm{r}$ \\
\hline 1127.579 & 73.08 & 93.08 & $\begin{array}{l}\text { TLDEFTIIQNLQPQ } \\
\text { YQFR }\end{array}$ & $\begin{array}{l}\text { Complement } \\
\text { subcomponent }\end{array}$ & $\mathrm{C} 1 \mathrm{r}$ \\
\hline 613.8062 & 38 & 58 & ALDFAVGEYNK & Cystatin & \\
\hline 549.2562 & 30.453 & 50.453 & YYGYTGAFR & Lactotransferrin & \\
\hline 510.7591 & 32 & 52 & DGAGDVAFIR & Lactotransferrin & \\
\hline 598.3089 & 37.635 & 57.635 & FQLFGSPSGQK & Lactotransferrin & \\
\hline 695.8699 & 49.105 & 69.105 & GGSFQLNELQGLK & Lactotransferrin & \\
\hline 565.3037 & 68.824 & 88.824 & EDAIWNLLR & Lactotransferrin & \\
\hline 1036.023 & 70.699 & 90.699 & $\begin{array}{l}\text { ADAVTLDGGFIYE } \\
\text { AGLAPYK }\end{array}$ & Lactotransferrin & \\
\hline 593.3148 & 19.275 & 39.275 & QNQIAVDEIR & Myeloperoxidase & \\
\hline 723.8466 & 26.089 & 46.089 & FPTDQLTPDQER & Myeloperoxidase & \\
\hline 548.8273 & 35.976 & 55.976 & QALAQISLPR & Myeloperoxidase & \\
\hline 640.8823 & 51.88 & 71.88 & VVLEGGIDPILR & Myeloperoxidase & \\
\hline 775.8941 & 22.421 & 42.421 & $\begin{array}{l}\text { GLDYASQQGTAAL } \\
\text { QK }\end{array}$ & $\begin{array}{l}\text { Bactericidal } \\
\text { permeability-increasing } \\
\text { protein }\end{array}$ & \\
\hline
\end{tabular}




\begin{tabular}{|c|c|c|c|c|}
\hline $\begin{array}{l}\text { Mass } \\
{[\mathrm{m} / \mathrm{z}]}\end{array}$ & $\begin{array}{l}\text { Start } \\
{[\min ]}\end{array}$ & $\begin{array}{l}\text { End } \\
{[\min ]}\end{array}$ & Peptide & protein \\
\hline 540.2902 & 26.343 & 46.343 & LQGSFDVSVK & $\begin{array}{l}\text { Lipopolysaccharide-binding } \\
\text { protein }\end{array}$ \\
\hline 624.3352 & 63.896 & 83.896 & ITLPDFTGDLR & $\begin{array}{l}\text { Lipopolysaccharide-binding } \\
\text { protein }\end{array}$ \\
\hline 599.3657 & 68.066 & 88.066 & LAEGFPLPLLK & $\begin{array}{l}\text { Lipopolysaccharide-binding } \\
\text { protein }\end{array}$ \\
\hline 1037.071 & 76.335 & 96.335 & $\begin{array}{l}\text { GLQYAAQEGLLAL } \\
\text { QSELLR }\end{array}$ & $\begin{array}{l}\text { Lipopolysaccharide-binding } \\
\text { protein }\end{array}$ \\
\hline 628.3125 & 2 & 22 & FVGGAENTAHPR & $\begin{array}{l}\text { Insulin-like growth } \\
\text { factor-binding protein }\end{array}$ \\
\hline 542.8217 & 56.498 & 76.498 & ALAAVLEELR & Pentraxin-related protein \\
\hline 715.8961 & 69.352 & 89.352 & LTSALDELLQATR & Pentraxin-related protein \\
\hline 1079.561 & 58.013 & 78.013 & $\begin{array}{l}\text { SFTLASSETGVGAP } \\
\text { ISGPGIPGR }\end{array}$ & Chitinase-3-like protein 1 \\
\hline 812.8655 & 29.114 & 49.114 & $\begin{array}{l}\text { GNQWVGYDDQES } \\
\text { VK }\end{array}$ & Chitinase-3-like protein 1 \\
\hline 489.2688 & 22.46 & 42.46 & SSFVAPLEK & epithelium-derived \\
\hline 528.3266 & 25.093 & 45.093 & TVQAVLTVPK & $\begin{array}{l}\text { Pigment epithelium-derived } \\
\text { factor }\end{array}$ \\
\hline 607.835 & 27.066 & 47.066 & ELLDTVTAPQK & epithelium-derived \\
\hline 758.8437 & 56.759 & 76.759 & TSLEDFYLDEER & epithelium-derived \\
\hline 692.3432 & 52.488 & 72.488 & LQSLFDSPDFSK & $\begin{array}{l}\text { Pigment epithelium-derived } \\
\text { factor }\end{array}$ \\
\hline 978.4911 & 53.179 & 73.179 & $\begin{array}{l}\text { ALYYDLISSPDIHG } \\
\text { TYK }\end{array}$ & epithelium-derived \\
\hline 625.835 & 60.084 & 80.084 & DTDTGALLFIGK & $\begin{array}{l}\text { Pigment epithelium-derived } \\
\text { factor }\end{array}$ \\
\hline
\end{tabular}




\begin{tabular}{|c|c|c|c|c|}
\hline $\begin{array}{l}\text { Mass } \\
{[\mathrm{m} / \mathrm{z}]}\end{array}$ & $\begin{array}{l}\text { Start } \\
{[\min ]}\end{array}$ & $\begin{array}{l}\text { End } \\
{[\mathrm{min}]}\end{array}$ & Peptide & protein \\
\hline 506.7278 & 6.8 & 26.8 & WESGYNTR & Lysozyme C \\
\hline 491.2229 & -0.099 & 19.901 & ATNYNAGDR & Lysozyme C \\
\hline 700.8439 & 48.998 & 68.998 & STDYGIFQINSR & Lysozyme C \\
\hline 513.3087 & 28 & 48 & IGGIGTVPVGR & Elongation factor 1 -alpha 1 \\
\hline 488.2791 & 29 & 49 & LPLQDVYK & Elongation factor 1 -alpha1 \\
\hline 702.8672 & 35 & 55 & YYVTIIDAPGHR & Elongation factor 1 -alpha 1 \\
\hline 657.8744 & 41 & 61 & EHALLAYTLGVK & Elongation factor 1 -alpha 1 \\
\hline 895.9446 & 32.391 & 52.391 & $\begin{array}{l}\text { VPLQQNFQDNQFQ } \\
\text { GK }\end{array}$ & $\begin{array}{l}\text { Neutrophil } \\
\text { gelatinase-associated lipocalin }\end{array}$ \\
\hline 628.3377 & 52.531 & 72.531 & SYPGLTSYLVR & $\begin{array}{l}\text { Neutrophil } \\
\text { gelatinase-associated lipocalin }\end{array}$ \\
\hline 716.409 & 68.868 & 88.868 & WYVVGLAGNAILR & $\begin{array}{l}\text { Neutrophil } \\
\text { gelatinase-associated lipocalin }\end{array}$ \\
\hline 706.3442 & 19.001 & 39.001 & $\begin{array}{l}\text { GDIGETGVPGAEG } \\
\text { PR }\end{array}$ & Adiponectin \\
\hline 886.4054 & 10.752 & 30.752 & $\begin{array}{l}\text { IFYNQQNHYDGST } \\
\text { GK }\end{array}$ & Adiponectin \\
\hline 532.803 & 37.923 & 57.923 & LALASLGYEK & $\begin{array}{l}\text { Programmed cell death } \\
\text { 6-interacting protein }\end{array}$ \\
\hline 765.8648 & 56.055 & 76.055 & $\begin{array}{l}\text { LANQAADYFGDAF } \\
\mathrm{K}\end{array}$ & $\begin{array}{l}\text { Programmed cell death } \\
\text { 6-interacting protein }\end{array}$ \\
\hline 864.4805 & 60.98 & 80.98 & $\begin{array}{l}\text { SVIEQGGIQTVDQL } \\
\text { IK }\end{array}$ & $\begin{array}{l}\text { Programmed cell death } \\
\text { 6-interacting protein }\end{array}$ \\
\hline 698.8772 & 68.787 & 88.787 & FYNELTEILVR & $\begin{array}{l}\text { Programmed cell death } \\
6 \text {-interacting protein }\end{array}$ \\
\hline 574.3215 & 47.189 & 67.189 & VFIGINDLEK & Collectin-11 \\
\hline 862.9407 & 71 & 91 & $\begin{array}{l}\text { EFADSLGIPFLETS } \\
\text { AK }\end{array}$ & Ras-related protein Rab-1B \\
\hline 565.3344 & 47.421 & 67.421 & VAFPLEVVQK & Interleukin-1 \\
\hline
\end{tabular}




\begin{tabular}{llllll}
\hline $\begin{array}{l}\text { Mass } \\
{[\mathrm{m} / \mathrm{z}]}\end{array}$ & $\begin{array}{l}\text { Start } \\
{[\mathrm{min}]}\end{array}$ & $\begin{array}{l}\text { End } \\
{[\mathrm{min}]}\end{array}$ & Peptide & protein & \\
\hline & & & & accessory protein & \\
577.8138 & 38.626 & 58.626 & LYIEYGIQR & $\begin{array}{l}\text { Interleukin-1 } \\
\text { accessory protein }\end{array}$ & receptor \\
666.3331 & 30.115 & 50.115 & QIQVFEDEPAR & $\begin{array}{l}\text { Interleukin-1 } \\
\text { accessory protein }\end{array}$ & receptor \\
& & & Cathepsin F & \\
636.7908 & 34.863 & 54.863 & FSDLTEEEFR & & \\
\hline
\end{tabular}


Table S4. 12 proteins successfully quantified in targeted proteomics

\begin{tabular}{|c|c|c|c|c|}
\hline Uniprot & Gene & Protein & $\begin{array}{l}\text { Difference value } \\
\text { (log2 transformed } \\
\text { ratio) }\end{array}$ & $\mathrm{P}$ value \\
\hline Q9UBX1 & CTSF & Cathepsin F & 0.078057 & 0.828112 \\
\hline Q9BWP8 & COLEC11 & Collectin-11 & 0.117387 & 0.590495 \\
\hline Q15848 & ADIPOQ & Adiponectin & -0.57841 & 0.155175 \\
\hline P61626 & LYZ & Lysozyme C & -0.19947 & 0.415894 \\
\hline P36955 & SERPINF1 & $\begin{array}{l}\text { Pigment } \\
\text { epithelium-derived factor }\end{array}$ & 0.463306 & 0.10491 \\
\hline P24593 & IGFBP5 & $\begin{array}{l}\text { Insulin-like growth } \\
\text { factor-binding protein } 5\end{array}$ & 0.077105 & 0.838939 \\
\hline P18428 & LBP & $\begin{array}{l}\text { Lipopolysaccharide-bindin } \\
\text { g protein }\end{array}$ & 1.166964 & 0.004044 \\
\hline P05164 & MPO & Myeloperoxidase & 0.514653 & 0.282458 \\
\hline P02788 & LTF & Lactotransferrin & 0.050411 & 0.915631 \\
\hline P01034 & CST3 & Cystatin-C & -0.13394 & 0.647523 \\
\hline P00736 & C1R & $\begin{array}{l}\text { Complement } \\
\text { subcomponent }\end{array}$ & 0.458279 & 0.04439 \\
\hline P00734 & $\mathrm{F} 2$ & Prothrombin & -0.41219 & 0.040723 \\
\hline
\end{tabular}


Table S5. 15 co-eluted and co-fragmented proteins in targeted proteomics

\begin{tabular}{|c|c|c|c|c|}
\hline Uniprot & Gene & Protein & $\begin{array}{l}\text { Difference value } \\
(\log 2 \text { ratio) }\end{array}$ & $\mathrm{P}$ value \\
\hline P02751 & FN1 & Fibronectin & -1.02554 & 0.035005 \\
\hline P04275 & VWF & von Willebrand factor & 1.002952 & 0.008476 \\
\hline P10909 & CLU & Clusterin & -0.556 & 0.010911 \\
\hline P19320 & VCAM1 & $\begin{array}{l}\text { Vascular cell adhesion } \\
\text { protein } 1\end{array}$ & 1.283055 & 0.00037 \\
\hline P07996 & THBS1 & Thrombospondin-1 & -0.98809 & 0.009884 \\
\hline P04180 & LCAT & $\begin{array}{l}\text { Phosphatidylcholine-sterol } \\
\text { acyltransferase }\end{array}$ & -0.82559 & 0.022164 \\
\hline P05156 & CFI & Complement factor I & 0.52669 & 0.026869 \\
\hline P19827 & ITIH1 & $\begin{array}{l}\text { Inter-alpha-trypsin inhibitor } \\
\text { heavy chain H1 }\end{array}$ & -0.85989 & 0.009734 \\
\hline P55058 & PLTP & Phospholipid transfer protein & 0.577778 & 0.027643 \\
\hline P02741 & CRP & $\begin{array}{l}\text { C-reactive protein [Cleaved } \\
\text { into: C-reactive protein }\end{array}$ & 1.830867 & 0.031885 \\
\hline P01011 & SERPINA3 & Alpha-1-antichymotrypsin & 0.91277 & 0.04947 \\
\hline P05546 & SERPIND1 & Heparin cofactor 2 & 0.547765 & 0.022252 \\
\hline P01024 & $\mathrm{C} 3$ & Complement C3 & 0.28393 & 0.16837 \\
\hline P02743 & APCS & $\begin{array}{l}\text { Serum amyloid P-component } \\
\text { (SAP) }\end{array}$ & -0.57962 & 0.050829 \\
\hline P05165 & PCCA & $\begin{array}{l}\text { Propionyl-CoA carboxylase } \\
\text { alpha chain }\end{array}$ & 0.41501 & 0.20143 \\
\hline
\end{tabular}


Table S6. Identification statistics for consensus database searching strategy.

\begin{tabular}{llll}
\hline & Run 1 & Run 2 & Run 3 \\
\hline total protein ID* & 1240 & 1226 & 1272 \\
Unique peptide IDs & 9314 & 9114 & 8653 \\
MS/MS acquired & 493791 & 478788 & 478273 \\
MS/MS identified & 62643 & 65181 & 65562 \\
MS/MS identified [\%] & 12.7 & 13.6 & 13.7 \\
Peptide sequences identified & 16200 & 17534 & 16598 \\
Average absolute mass deviation [+-ppm] & +0.73 & +0.72 & +0.73 \\
\hline
\end{tabular}

Note: * protein identified at $\mathrm{FDR}<1 \%$, with two unique peptides. 
Table S7. 9 potential urosepsis diagnostic biomarkers with auROC $>0.7$

\begin{tabular}{lllll}
\hline & Sen & Spe & AUC & p value \\
\hline LBP & 70 & 90.5 & 0.75 & 0.004044 \\
VCAM1 & 75 & 81 & 0.8 & 0.00037 \\
CRP & 60 & 85.7 & 0.724 & 0.031885 \\
CLU & 55 & 90.5 & 0.717 & 0.010911 \\
VWF & 70 & 81 & 0.76 & 0.008476 \\
THBS1 & 65 & 76.2 & 0.738 & 0.009884 \\
FN1 & 75 & 71.4 & 0.724 & 0.035005 \\
SERPINA3 & 50 & 100 & 0.706 & 0.04947 \\
ITIH1 & 65 & 76.2 & 0.7 & 0.009734 \\
\hline
\end{tabular}


Table S8. Logistic model with 4 proteins and its statistics

\begin{tabular}{llll}
\hline Variable & Coefficient & Std. Error & $\mathrm{P}$ \\
\hline LBP & 3.317 & 1.642 & 0.043 \\
VCAM1 & 2.749 & 1.192 & 0.021 \\
CLU & -5.169 & 2.105 & 0.014 \\
CRP & -1.230 & 0.651 & 0.059 \\
Constant & 40.721 & & \\
\hline
\end{tabular}

Table S9. Logistic model with 3 proteins and its statistics

\begin{tabular}{llll}
\hline Variable & Coefficient & Std. Error & $\mathrm{P}$ \\
\hline LBP & 0.588 & 0.446 & 0.187 \\
VCAM1 & 2.059 & 0.768 & 0.007 \\
CLU & -3.074 & 1.195 & 0.010 \\
Constant & 34.688 & & \\
\hline
\end{tabular}


Table S10. Kidney injury associated DEPs in the discovery TMT datasets

\begin{tabular}{cccccccc}
\hline & & \multicolumn{2}{c}{ N2_S2 } & \multicolumn{2}{c}{ N4_S4 } & \multicolumn{2}{c}{ N6_S6 } \\
gene & protein & $-\log p$ & FC & $-\log p$ & FC & $-\log p$ & FC \\
\hline CLU & Clusterin & $\mathbf{1 . 3 9 4}$ & 1.208 & 1.165 & 2.026 & 1.054 & 1.625 \\
CST3 & Cystatin-C & $\mathbf{4 . 9 3 6}$ & 0.633 & $\mathbf{2 . 0 4 4}$ & 0.584 & $\mathbf{1 . 9 7 5}$ & 0.686 \\
KIF12(AMBP) & Alpha-1-microglobulin & 0.837 & 0.814 & 0.111 & 0.945 & 0.084 & 0.973 \\
AHSG & Alpha-2-HS-glycoprotein & 0.323 & 1.075 & 0.176 & 1.105 & $\mathbf{2 . 0 7 4}$ & 0.693 \\
\hline
\end{tabular}

fold changes $(\mathrm{FC})$ were calculated by S/N. Pvalue were log10 transformed. 